\title{
On the Single Wall Carbon Nanotube Surface Plasmon Stability
}

\author{
Jaime Silva ${ }^{1, *}$, Bruce F. Milne ${ }^{1, * *}$, and Fernando Nogueira ${ }^{1, * * *}$ \\ ${ }^{1}$ CFisUC, Department of Physics, University of Coimbra, Rua Larga, 3004-516 Coimbra, Portugal.
}

\begin{abstract}
The physics of surface plasmons has a long tradition in condensed matter theory but as the dimension of the systems reaches the nano scale, new effects appear. In this work, by calculating the absorption spectra of a single wall carbon nanotube, using time dependent density functional theory, the effect of adding/removing electrons on the surface plasmon energy is studied. It is shown that removing electrons from the single wall carbon nanotube does not affect the surface plasmon energy peak. In contrast, adding electrons to the single wall carbon nanotube will redshift the plasmonic peak energy, an effect that is explained by an increase of the electron effective mass.
\end{abstract}

\section{Introduction}

The surface plasmon on metals has a long history that started with the explanation of experimental results by Pines and Bohm [1-3]. Pines and Bohm explained that the longitudinal oscillation of the electron density observed in thin metal films when bombarded with fast electrons is due to long-range coulomb interaction of the valence electrons in the metal films [1-3]. The latter authors coined the term "plasmon" to describe the collective oscillation of the electron density due to the similarity with the electronic plasma oscillations in gas discharges [1-3]. Finally, Stern and Ferrell [4] also described these collective oscillations of the density as surface plasmons.

It is important to distinguish between bulk and surface plasmons. Using classic electrodynamics the bulk plasmon dispersion relation can be retrieved by solving $\nabla \cdot \mathbf{D}=0$ for field propagating in a medium with no sources, giving two solutions. The first solution of the latter equation is the bulk plasmon and second is the dispersion relation for photons propagating in a medium which is called a polariton $[1,5]$. The surface plasmon dispersion relation is obtained following a similar procedure but solving the $\nabla \cdot \mathbf{D}=0$ for electric fields propagating in the upper and lower half-spaces of the surface.

As the dimension of the system is reduced, a quantum description of the surface plasmon is needed. The field that studies the plasmonic oscillations using quantum mechanics is termed quantum plasmonics [6]. The use of quantum mechanics to describe the surface plasmons comes from the fact that classical electrodynamics fails to describe plasmonic resonances at sub-nanometer gaps [7]. In fact, Drude's model is usually employed to describe

\footnotetext{
*e-mail: jaime.d.silva@uc.pt

**e-mail: bfmilne@uc.pt

***e-mail: fnog@uc.pt
} 
the surface plasmons of small nanoparticles. Nevertheless, Drude's model fails in the prediction of the blueshift of the plasmonic peak energy when decreasing the nanoparticle size [8]. The latter is due to the fact that for small particles the dielectric function cannot describe a small molecule. On way to overcome the limitations of the classic approach is to use time dependent density functional theory (TDDFT) [9, 10]. Using TDDFT, Zuloaga et. al. [11] found that the optical spectra is very different from the ones obtained using classical calculations. The latter authors concluded that the electron spill-out at the surface induces a screening that is not captured using a classical calculation. Townsend et. al. [12], using TDDFT, characterised the resonances of metallic nanoparticles investigating which characteristics are important to decide if a resonance is plasmonic. In another related work, Yan et. al. [13], using TDDFT, demonstrated for a linear atomic chain the existence of end and central plasmon resonances. The existence of plasmon resonances at the nanoscale opens the route for plasmonic nanosensors. In fact, as discussed in [14], this type of sensors are able to detect single molecules. Furthermore, the existence of surface plasmon resonance modes in nanoparticles enables their use as biochemical sensors, among other applications [14]. The observation of a surface plasmon in the optical response of single-wall nanotubes (SWCNT) [15] can pave the way for the construction of new type SWCNT plasmonic sensors with a vast application range. In a previous work [16] the plasmonic peak in the optical spectra of SWCNT was studied in detail. The purpose of this work is to study, theoretically, the stability of the surface plasmon seen in metallic carbon nanotubes [16] upon electron addition, removal and adsorption of organic gases.

This work is organized as follows. In the methodology section we present the details of the theoretical calculation. This is followed by the results and discussion section and the main conclusions.

\section{Methodology}

In this work we use density functional theory (DFT)[17] and TDDFT to calculate[18] the absorption spectra, the paramagnetic current and the density of states (DOS) of a (3,3) SWCNT with removed/added electrons. In the framework of DFT/TDDFT the Hamiltonian is given by Equation 1.

$$
\hat{H}=\frac{1}{2}\left(-i \nabla+\frac{1}{c} \mathbf{A}_{e x t}\right)^{2}+\hat{v}
$$

where $\mathbf{A}_{\text {ext }}$ is the external vector potential. For an uniform static magnetic field $\mathbf{B}$ applied to a finite system with zero total spin, the vector potential, $\mathbf{A}$, can be defined using the Coulomb gauge by:

$$
\mathbf{A}(\mathbf{r})=\frac{1}{2} \mathbf{B} \times \mathbf{r}
$$

The time-dependent Kohn-Sham (KS) orbitals are determined using the Hamiltonian of Equation 1 where $\hat{v}$ is the KS effective potential that can be decomposed in an external potential, an Hartree potential and an exchange-correlation potential. In our calculations we used a uniform real space grid to discretise the equations. Spacing was $0.20 \AA$ and the simulation box was constructed by joining spheres of radius $3.5 \AA$ around every atom. For the time domain, a time step of 0.000658 fs was employed for the propagation of the KS equations. Finally, the exchange-correlation potential was approximated by using the local density approximation. 
In this work, the finite SWCNT were generated ${ }^{1}$ with 15 translations of the unit cell along the tube orientation and the terminations of the finite SWCNT were passivated with hydrogens. Finally, all the structures were optimised within DFT using the exchange functional of Tao et el (TPSS) [19] and using Def2-TZVP basis sets with Grimme's D3 dispersion corrections, as provided by the ORCA package[20].

\section{Results and discussion}

As the size of the system diminishes, the stability of the surface plasmon upon electron removal and addition becomes increasingly important for the final electronic design of the device. In Figure 1 we present the theoretical spectra of a SWCNT when pairs of electrons are removed from it.

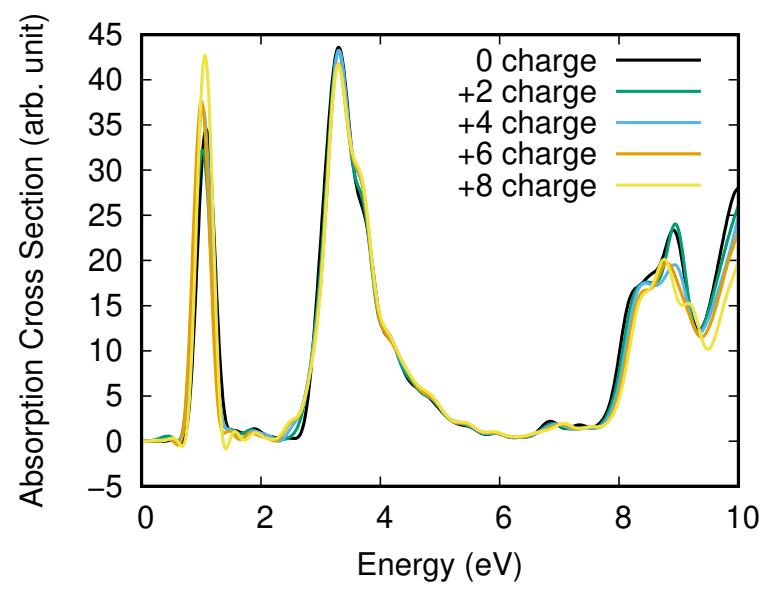

Figure 1. Absorption spectra of a SWCNT when electrons are removed from it.

It is possible to observe in Figure 1 that the plasmonic peak[16] energy, the first one, remains the same when the electrons are removed. In fact, by observing the density of states (DOS) of SWCNT, Figure 3, it can be shown that it remains the same. But absorption spectra peak heights depend on the number of electrons in each system.

In Figure 2 we present the absorption spectra for a $(3,3)$ SWCNT with added electrons. As can be seen in the latter figure, adding electrons to the SWCNT will redshift the surface plasmon peak.

This redshift can be understood by looking at the DOS presented in Figure 4, where it can be seen that adding electrons to a SWCNT will redshift the DOS. Hence, this shift in DOS will induce the observed shift in the absorption spectra. Interestingly, the DOS does not change when electrons are removed, as can be seen in Figure 3.

A possible explanation for the change in the DOS upon electron addition is the increase of the effective mass of the electrons[21] due to Coulomb repulsion, that will reduce the excitation energy for low bounded electrons. It should be stressed that in phenomenological models of [21] the plasmonic regime, a critical density that depends on the motion of electrons dictated by Coulomb repulsion rather than a confinement of the carriers is achieved. It is

\footnotetext{
${ }^{1}$ TubeGen 3.4 (web-interface, http://turin.nss.udel.edu/research/tubegenonline.html), J. T. Frey and D. J. Doren, University of Delaware, Newark DE, 2011
} 


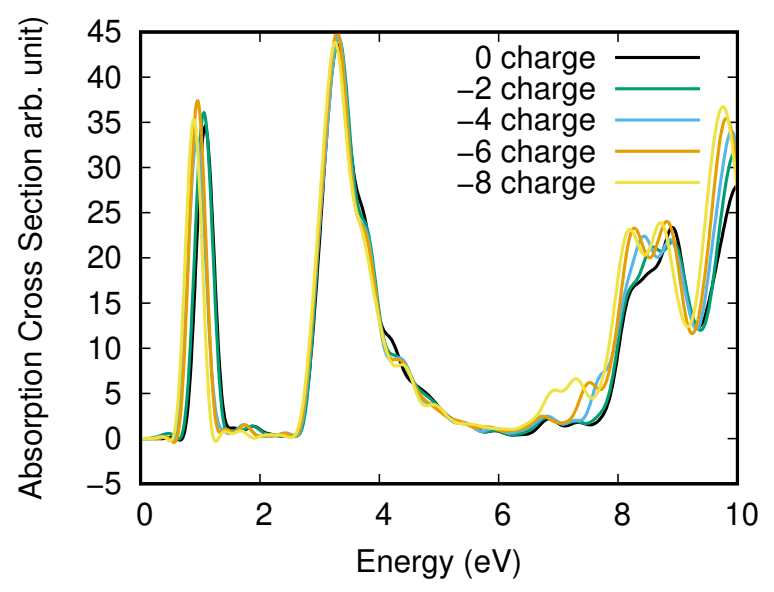

Figure 2. Absorption spectra of a SWCNT when electrons are added to it.

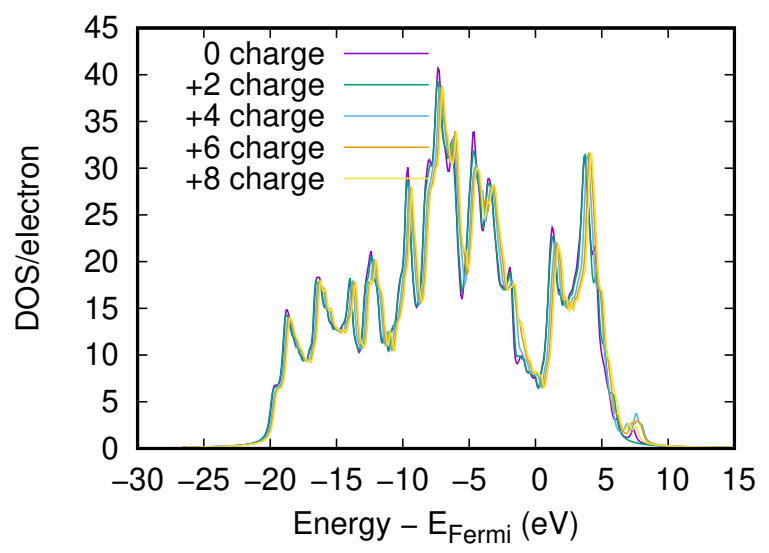

Figure 3. DOS for a SWCNT when electrons are removed from it.

possible to observe in their work a redshift of the plasmon peak with the increase of the carrier effective mass. One point that should be discussed regarding the model of [21] is that, for the latter model, adding carriers to a system will blueshift the energy of the surface plasmon, a behaviour contrary to what is observed in this work. This can be explained by the high electron density of the metallic SWCNT, that remains practically unchanged with the addition of a few electrons.

Taking in mind the last observation, we concluded that the electrons added to the SWCNT will not participate directly in the collective resonance that forms the surface plasmon, but will contribute to an increase of the SWCNT electron effective mass that, as discussed in [21], will redshift the surface plasmon energy. 


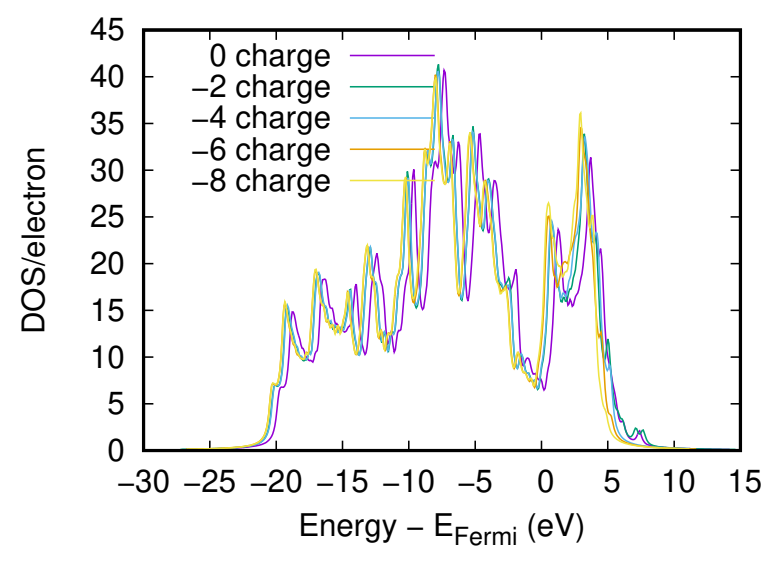

Figure 4. DOS for a SWCNT when electrons are added to it.

\section{Conclusion}

In this work we study the stability of the surface plasmon upon electron removal/addition. We demonstrate that removing electrons from a $(3,3)$ SWCNT does not affect the plasmonic peak energy present in the SWCNT absorption spectrum. But electron addition will induce a redshift in the plasmonic peak energy, correlated to a redshift of the DOS. This redshift of the plasmonic energy peak with the addition of electrons can be related to the increase of the electron effective mass that, due to Coulomb repulsion, reduces the excitation energy for low bounded electrons.

\section{Acknowledgments}

We thank the Laboratory for Advanced Computing (LCA) of the University of Coimbra for the computer time allocated to this project.

\section{References}

[1] I.R. Hooper, W.L. Barnes, in Handbook of Surface Science (Elsevier B.V., 2014), Vol. 4, pp. 34-74

[2] D. Pines, D. Bohm, Phys. Rev. 85, 338 (1952)

[3] D. Pines, Rev. Mod. Phys. 28, 184 (1956)

[4] E.A. Stern, R.A. Ferrell, Phys. Rev. 120, 130 (1960)

[5] J.J. Hopfield, Phys. Rev. 112, 1555 (1958)

[6] M.S. Tame, K.R. McEnery, S.K. Ozdemir, J. Lee, S.A. Maier, M.S. Kim, Nat. Phys. 9, 329 (2013)

[7] R. Esteban, A.G. Borisov, P. Nordlander, J. Aizpurua, Nat. Commun. 3, 825 (2012)

[8] E.B. Guidez, C.M. Aikens, Nanoscale 6, 11512 (2014)

[9] E. Runge, E.K.U. Gross, Phys. Rev. Lett. 52, 997 (1984)

[10] M. Marques, N. Maitra, F. Nogueira, E.K.U. Gross, A. Rubio, Fundamentals of TimeDependent Density Functional Theory, Lecture Notes in Physics (Springer Berlin Heidelberg, 2012) 
[11] J. Zuloaga, E. Prodan, P. Nordlander, ACS Nano 4, 5269 (2010)

[12] E. Townsend, G.W. Bryant, J. Optics-Uk 16, 114022 (2014)

[13] J. Yan, Z. Yuan, S. Gao, Phys. Rev. Lett. 98, 216602 (2007)

[14] J.N. Anker, W.P. Hall, O. Lyandres, N.C. Shah, J. Zhao, R.P. Van Duyne, Nat. Mater. 7, 442 (2008)

[15] T. Morimoto, S.K. Joung, T. Saito, D.N. Futaba, K. Hata, T. Okazaki, ACS Nano 8, 9897 (2014)

[16] J. Silva, M.J.T. Oliveira, S. Lanceros-Mendez, F. Nogueira, J. Phys. Chem. C 120, 18268 (2016)

[17] P. Hohenberg, W. Kohn, Phys. Rev. 136, B864 (1964)

[18] X. Andrade, D. Strubbe, U. De Giovannini, A.H. Larsen, M.J.T. Oliveira, J. AlberdiRodriguez, A. Varas, I. Theophilou, N. Helbig, M.J. Verstraete et al., Phys. Chem. Chem. Phys. 17, 31371 (2015)

[19] J. Tao, J.P. Perdew, V.N. Staroverov, G.E. Scuseria, Phys. Rev. Lett. 91, 146401 (2003)

[20] F. Neese, Wires Comput. Mol. Sci. 2, 73 (2012)

[21] P.K. Jain, J. Phys. Chem. Lett. 5, 3112 (2014) 